\title{
Análise Experimental dos Modos de Balanceamento de Réplicas para o HDFS Balancer
}

\author{
Rhauani Weber Aita Fazul ${ }^{1}$, Patrícia Pitthan Barcelos ${ }^{1}$ \\ ${ }^{1}$ Pós-Graduação em Ciência da Computação (PGCC) \\ Universidade Federal de Santa Maria (UFSM) \\ Santa Maria - RS - Brasil \\ \{rwfazul, pitthan\}@inf.ufsm.br
}

\begin{abstract}
Resumo. O HDFS Balancer é a ferramenta nativa para o balanceamento de réplicas no HDFS. Ao disparar a daemon do balanceador, o administrador do sistema deve definir as configurações a serem utilizadas. De forma a auxiliar a tomada de decisão, este trabalho avalia a aplicabilidade do HDFS Balancer com as configurações recomendadas para três modos de balanceamento: default, background e fast. Os resultados demostram a efetividade de cada modo.
\end{abstract}

\section{Introdução}

O Hadoop Distributed File System (HDFS), motor de armazenamento do Apache Hadoop, é um sistema de arquivos escalável e altamente confiável. Quando um arquivo é inserido no HDFS, esse é segmentado em blocos de dados de tamanho fixo, que são replicados e armazenados em múltiplos DataNodes. O posicionamento das réplicas através do cluster é essencial para o funcionamento adequado do HDFS, já que uma distribuição desequilibrada afeta a localidade dos dados e o desempenho do sistema.

Dentre as principais causas do desbalanceamento estão [Hortonworks 2019]: (i) a Política de Posicionamento de Réplicas empregada pelo HDFS; (ii) o comportamento da aplicação do cliente; (iii) o processo de re-replicação; e (iv) a adição de novos DataNodes ao sistema. A solução oficial, integrada na distribuição do Hadoop, para mitigar os problemas inerentes do desbalanceamento de réplicas é o HDFS Balancer [White 2015].

Este trabalho analisa a aplicabilidade de diferentes configurações para o HDFS Balancer, considerando a execução da ferramenta baseada em diferentes modos de balanceamento. Uma investigação experimental foi conduzida a fim de evidenciar o comportamento de cada modo e seus impactos na operação de balanceamento de réplicas.

O trabalho está organizado em quatro seções. A Seção 2 apresenta o HDFS Balancer e descreve os modos de balanceamento. A Seção 3 exibe os resultados obtidos na experimentação. Por fim, a Seção 4 conclui o artigo e direciona os trabalhos futuros.

\section{HDFS Balancer}

O HDFS Balancer é uma ferramenta destinada ao balanceamento de réplicas entre dispositivos de armazenamento no HDFS [White 2015]. A partir da análise do posicionamento dos blocos, o balanceador toma decisões referentes à redistribuição dos dados, fazendo com que a utilização dos DataNodes fique dentro de um intervalo controlado.

Além de disparar a execução do HDFS Balancer, o administrador do sistema deve definir as configurações do balanceador. Pensando nos diferentes contextos em que 
a operação é realizada, [Hortonworks 2019] detalha três modos de balanceamento com valores recomendados para as propriedades de configuração do HDFS Balancer, sendo esses: (i) default, no qual a ferramenta executa com as configurações padrão; (ii) background, em que o balanceador opera como um processo em segundo plano, visando reduzir o impacto nos demais jobs e aplicações no cluster; e (iii) fast, no qual o HDFS Balancer atua para que o processo de balanceamento seja concluído o mais rápido possível.

\section{Experimentação}

A experimentação foi realizada na plataforma GRID' 5000 ${ }^{1}$ com o Hadoop (versão 2.9.2) operando em um ambiente com 10 nodos, cada um com 2 CPUs Intel Xeon E5-2630 v3 (8 cores/CPU), 128GB RAM, 558GB HDD e 4 conexões Ethernet de 10Gb. O benchmark TestDFSIO [White 2015] foi utilizado para escrever 30 arquivos de 30GB com um fator de replicação padrão de 3, totalizando um volume de 2,66TB. A Tabela1 exibe os resultados considerando os valores médios de 20 operações do HDFS Balancer após a carga dos dados com cada um dos modos de balanceamento e um threshold padrão de $10 \%$.

Tabela 1. Uso do balanceador com os diferentes modos de balanceamento.

\begin{tabular}{lccc}
\hline \multicolumn{1}{c}{ Métrica } & Default & Background & Fast \\
\hline Volume de dados movimentado & $204,75 \mathrm{~GB}$ & $211,88 \mathrm{~GB}$ & $215,50 \mathrm{~GB}$ \\
Iterações de balanceamento & 14 & 82 & 7 \\
Tempo de execução & $2 \mathrm{~h} 44 \mathrm{~m} 27 \mathrm{~s}$ & $3 \mathrm{~h} 11 \mathrm{~m} 18 \mathrm{~s}$ & $14 \mathrm{~m} 29 \mathrm{~s}$ \\
Consumo de largura de banda & $20,75 \mathrm{MB} / \mathrm{s}$ & $18,46 \mathrm{MB} / \mathrm{s}$ & $247,99 \mathrm{MB} / \mathrm{s}$ \\
\hline
\end{tabular}

Percebe-se como os modos de balanceamento causam diferenças significativas na operação do balanceador. A variação percentual do tempo de execução do modo background em relação ao default, foi de 16,33\%, indicando o aumento ocasionado. Com o modo fast, por sua vez, a variação foi de $-91,19 \%$, indicando a redução. Todavia, enquanto os modos background e default limitam de forma rigorosa a utilização dos recursos, o modo fast opera sem preocupação em causar sobrecarrega, com mais threads para a movimentação dos blocos e consumindo uma maior largura de banda do cluster.

\section{Considerações Finais}

Este trabalho analisou o uso dos modos de balanceamento default, background e fast para o HDFS Balancer. Os experimentos evidenciam o trade-off entre a rapidez e o custo da operação de cada modo. Baseado nisso, espera-se auxiliar a tomada de decisão de administradores de clusters HDFS para a configuração ideal do balanceador. Trabalhos futuros envolvem uma investigação aprofundada do comportamento do HDFS Balancer sob diferentes configurações, considerando diferentes cargas de trabalho e os custos envolvidos no processo de balanceamento. Além disso, pretende-se avaliar o impacto das diferentes Políticas de Posicionamento de Réplicas do HDFS durante a redistribuição dos dados.

\section{Referências}

Hortonworks (2019). "Balancing data across an HDFS cluster". https:// docs. cloudera.com/HDPDocuments/HDP 3/HDP-3.1.4/data-storage/ content/balancing_data_across_hdfs_cluster.html. Dezembro.

White, T. (2015). Hadoop: The Definitive Guide. O’Reilly Media, Inc., 4 edition.

1https://www.grid5000.fr 